\title{
Caudal regression associated with pre-gestational type 2 diabetes mellitus
}

\author{
S Raguramana, M Karunarathne ${ }^{\mathrm{b}}$, N Jayantha ${ }^{\mathrm{c}}$, S Bandara ${ }^{\mathrm{d}}$
}

\begin{abstract}
Caudal regression syndrome is a spectrum of congenital structural defects associated with uncontrolled pre-gestational diabetes mellitus. Prevalence of this disease is 1 to 2.5 in 100,000 live births and background pre-gestational diabetes increases the risk by 200 fold.

We present a case of caudal regression syndrome in a mother having type 2 diabetes mellitus with poor glycaemic control since preconceptional period and early gestation. The condition was diagnosed during anomaly scan at 20 weeks of gestation.

Uncontrolled pre-existing diabetes is the most common aetiology for caudal regression syndrome. Therefore, proper preconceptional counseling is essential to optimize blood sugar levels in pre-gestational diabetic women. Carrying out anomaly scans around 20 weeks play a pivotal role in high risk cases in diagnosing foetal anomalies.
\end{abstract}

Key words: Caudal regression syndrome, Pregestational diabetes, Sacral Agenesis, Anomaly scan

Sri Lanka Journal of Obstetrics and Gynaecology 2018; 40: 67-69

DOI: http://doi.org/10.4038/sljog.v40i3.7858

\section{Introduction}

Caudal regression syndrome is a spectrum of congenital structural defects associated with uncontrolled pre-gestational diabetes mellitus. Prevalence of disease is 1 to 2.5 in 100,000 live births ${ }^{1}$ and background pregestational diabetes increases the risk by 200 fold $^{2,3}$. Aetiology for this condition includes chromosomal anomalies, especially mutation of the HLXB9 gene (142994) on chromosome 7q36, vascular hypoperfusion, foetal hyperglycemia and in-utero exposure to drugs such as Trimetoprim-Sulpnamide. The spectrum extends from partial (unilateral deformation of sacrum), bilateral deformation and most severe types involving total absence of sacrum ${ }^{2}$. We present a rare case of caudal regression syndrome (CRS) in a mother with uncontrolled type 2 diabetes mellitus.

\section{Case report}

A 24 year old primi-gravid presented at 20 weeks of period of gestation for an anomaly scan. She had been diagnosed with type II diabetes mellitus for the duration of past 10 years. She was on medical nutritional therapy. She had poor drug compliance as well as had defaulted clinic follow-up in the pre-gestational period. During early gestation she was treated with metformin. However, her compliance on blood sugar monitoring and antenatal follow-up were also poor. At the time of

a Senior Registrar in Obstetrics \& Gynaecology,

${ }^{\mathrm{b}}$ Consultant Obstetrician \& Gynaecologist,

${ }^{c}$ Registrar in Obstetrics \& Gynaecology,

${ }^{\mathrm{d}}$ Registrar in Obstetrics \& Gynaecology, Sri Jayewardenepure General Hospital, Colombo, Sri Lanka.

Correspondence: RS, e-mail: <sivalingarajahraguraman@gmail.com>

http://orcid.org/0000-0001-7157-3680

Competing interest: The authors report no conflict of interest 
presentation, her HbA1C was 9.8\%. Post Prandial Blood Sugar was $240 \mathrm{mg} / \mathrm{dl}$ and 6 value blood sugar series showed poor glycemic control.

Anomaly scan revealed a demised foetus with consistent growth parameters for dates and an adequate amount of liquor. A detailed scan revealed sudden termination of spine at lumbar level and fixed lower extremities with club foot. It further showed relative atrophy of two legs, feet and sacrum with characteristic frog like limbs. Spinal cord had a closed neural tube defect and other organs including brain, liver lungs and heart appeared normal. She delivered a fetus weighing $650 \mathrm{~g}$ with a normal morphological placenta by medical induction. Pathological postmortem findings confirmed the caudal regression syndrome.

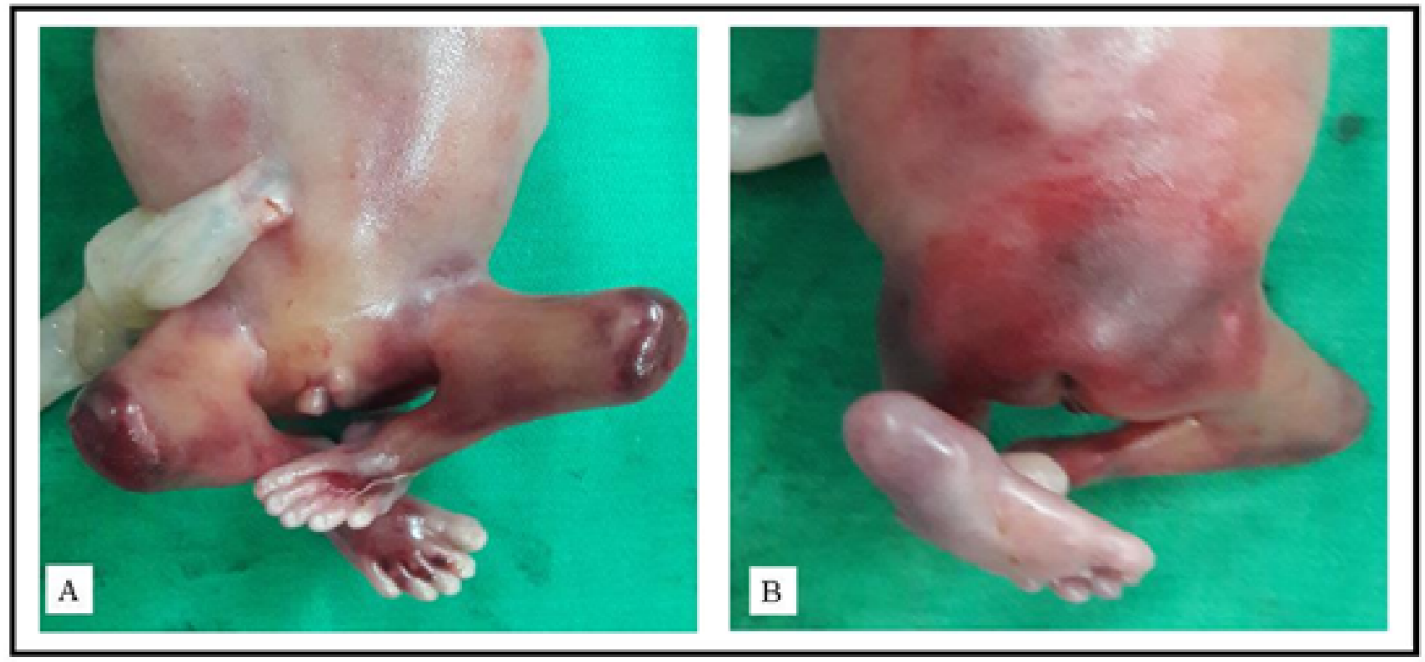

Figure 1. A - Limb hypoplasia

B - Pelvic and sacral hypoplasia

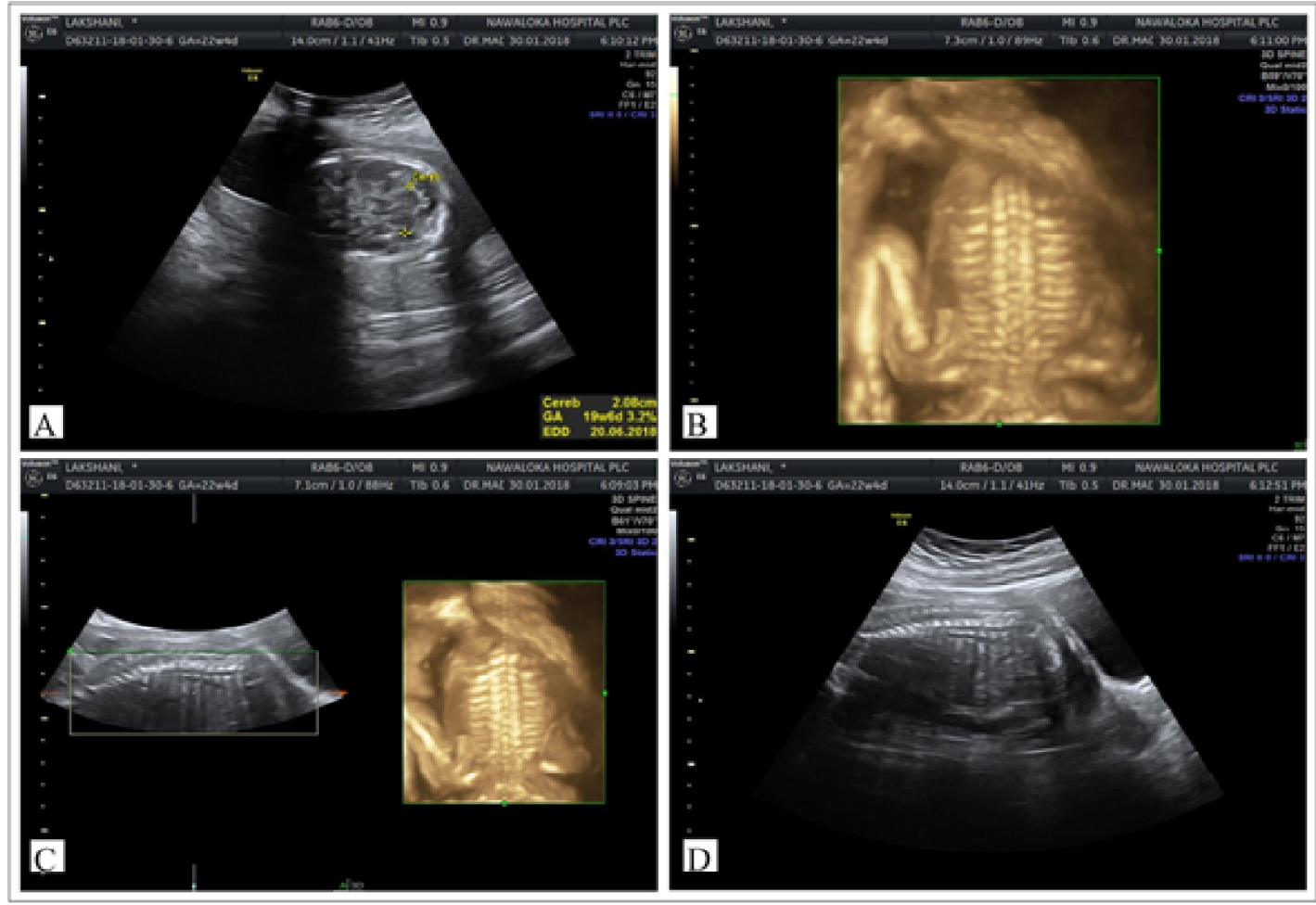

Figure 2. A - Skull normal

B/C/D - Pelvic and sacral hypoplasia 


\section{Discussion}

Prevalence of caudal agenesis syndrome is approximately 1 to 2.5 per 100,000 live births. There are multiple genetic and environmental risk factors playing a role in the aetiology. Maternal diabetes is a wellestablished causative factor. It is estimated that 1 in 350 babies are affected where mothers had uncontrolled diabetes mellitus. This condition arises during $3^{\text {rd }}$ to $7^{\text {th }}$ week of gestation due to abnormal gastrulation and disturbance of mesodermal migration.

Caudal regression is also known as sacral agenesis, caudal dysgenesis syndrome, caudal dysplasia sequence, caudal regression sequence, sacral agenesis and sacral defect with anterior meningocele ${ }^{2}$. It is a congenital disorder, in which there is abnormal development of the caudal part of the body. This includes lower back, lower limbs, genitourinary and gastrointestinal tract. Some may have abnormal curvatures such as scoliosis. Lower sacral vertebrae might be absent or misshaped with or without a neural tube defect.

Genitourinary defects are extremely varied. Ectopia vesicae, absent kidney, hypospediasis, cryptorchidism, recto-vaginal fistula are common and total genital agenesis might occur in extreme cases. Gastroenterological defects are abnormal development of the gut, malrotation of large intestine, inguinal hernia, imperforated anus and loss of control of bladder and bowel occurring in extreme cases. A permanent colostomy may be needed for imperforated anus. Occasionally surgical correction may be required for lower limbs deformities and amputation of the limbs in childhood may be needed in extreme cases.

Some evidence suggest that this disorder may occur due to abnormal arterial development of the abdomen with diversion of blood flow away from the developing caudal portion. Resultant hypoperfusion and hypoxeamia cause mal-development.

Prenatal diagnosis of this condition depends on the severity of maldevelopment. During anomaly scan it is important to visualize the entire length of the spine, sacral portion and lower limbs. Diagnosis is achieved visualizing the absence of lower lumbar vertebrae with the presence of lower limb abnormalities which were seen in this case. Magnetic resonance imaging may confirm the diagnosis in high resources settings. Usual diagnosis period is around $20-24$ weeks of pregnancy ${ }^{2,3}$.

Prognosis of the disease may depend on the severity of the defects. Hence complete prenatal diagnosis and parental counseling should be carried out prior to taking a joint decision on further management.

In our case, mother had pregestational uncontrolled diabetes mellitus with an $\mathrm{HbA} 1 \mathrm{c}$ of $9.8 \%$. The foetus was found to have absent lower lumbar and sacral vertebrae with incomplete development of lower limbs ${ }^{4}$. This case was diagnosed at 20 weeks of gestation and confirmed with a pathological post mortem.

\section{Conclusion}

Caudal regression syndrome is a multifactorial structural deformity of the foetus, which can be diagnosed prenatally. Since uncontrolled pre-exsisting diabetes is the most common aetiology, it is essential to carry out proper pre-conceptional counselling and achieve adequate glycaemic control prior to pregnancy in patients with chronic diabetes. Anomaly scan around 18 to 20 weeks of gestation plays a pivote role in diagnosing such cases in high risk mothers.

\section{References}

1. Semba K. Etiology of caudal Regression Syndrome. Hum genet Embryol. 2013; 03(02).

2. Temizkan $O$ prenatal diagnosed caudal regression syndrome. Open J Obstet Gynecol. 2013; 03(02) 227-231.

3. Sonek JD,Gabbe SG “Antenatal diagnosis of Sacral agenesis syndrome in a pregnancy complicated by diabetes mellitus" Am. J. Obstet, Gynecol 162(3); 806-8.

4. Pang d Sacral agenesis and caudal spinal cord malformations. Neurosurgery.193; 32: 755-78. 